nephron

Experimental

Nephrology

and Genetics
Nephron 2018;138:137-146

DOI: $10.1159 / 000481441$
Received: March 1, 2017

Accepted after revision: September 11, 2017

Published online: October 19, 2017

\title{
Reading First Coordinates from the Nephrogenic Zone in Human Fetal Kidney
}

\author{
Will W. Minuth \\ Institute of Anatomy, University of Regensburg, Regensburg, Germany
}

\section{Keywords \\ Human fetal kidney · Preterm infants - Low birth weight babies - Impaired nephrogenesis · Nephrogenic zone Stem cell niche $\cdot$ Coordinates $\cdot$ Microanatomy}

\begin{abstract}
While substantial information is available on organ anlage and the primary formation of nephrons, molecular mechanisms acting during the late development of the human kidney have received an astonishing lack of attention. In healthy newborn babies, nephrogenesis takes place unnoticed until birth. Upon delivery, morphogenetic activity in the nephrogenic zone decreases, and the stem cell niches aligned beyond the organ capsule vanish by an unknown signal. However, this signal also plays a key role in preterm and low birth weight babies. Although they are born in a phase of active nephrogenesis, pathological findings illustrate that they evolve to a high incidence oligonephropathy and prematurity of renal parenchyma. Different extra- and intrauterine influences seem to be responsible, but independent from chemical nature, all of them culminate in the nephrogenic zone. One assumes that the marred development is caused either by an overshoot of metabolites, misleading signaling of morphogens, unbalanced synthesis of extracellular matrix or restricted contact between mesenchymal and epithelial
\end{abstract}

stem cells. Even more surprising is that there is only a few vague morphological information of the nephrogenic zone in the human fetal kidney available and ultrastructural data is severely lacking. On this account, the first coordinates were determined by optical microscopy and morphometry. Without claiming to be complete, generated results made it possible to create schematic illustrations true to scale for orientation. It will help graduating students, young pediatricians, pathologists, and scientists working in the field of biomedicine to interpret professionally the nephrogenic zone and contained niches.

(c) 2017 S. Karger AG, Basel

The treatment of renal failure has changed little during the last decades. Dialysis and transplantation are still the sole therapeutic options for patients suffering from end stage renal disease. Lack of a cure is frequently attributed to a low intrinsic capacity of the kidney for self-repair [1]. Nevertheless, clinical experiences in past years demonstrate that some regeneration is possible [2]. Because of which, substantial efforts are being made to stimulate regeneration with the help of implanted stem cells [3-6]. To provide an effective source and an optimal course of regeneration, different kinds of stem cells were tested and various implantation techniques tried $[7,8]$. Although

\section{KARGER}

(c) 2017 S. Karger AG, Basel

E-Mail karger@karger.com

www.karger.com/nef
Prof. Dr. Will W. Minuth

Institute of Anatomy, University of Regensburg

University Street 31

DE-93053 Regensburg (Germany)

E-Mail will.minuth@ @kl.uni-regensburg.de 
some approaches looked promising, survival of implanted stem cells in the inflammatory environment of a diseased kidney is still unexpectedly limited to a few days [9].

To optimize survival, experiments are being performed on seeding stem cells before an implantation, for example, on organ-specific extracellular matrix [10-13]. Other than an implantation of isolated stem cells, research is following up with a biomedical activation of quiescent stem cells occurring in adult renal parenchyma [14-17]. An alternative approach is to investigate reactivation of earlier stem cell niches represented during entire organ development of the original source of renal parenchyma $[18,19]$.

To date, there is uncertainty about the molecular mechanism that keeps the stem cells dormant during kidney disease. However, a basic hint for the mechanism restricting stem cell activity can be perceived in developing kidneys of preterm and low birth weight babies [20]. Although they are delivered in a phase of active nephrogenesis, between 8 and $24 \%$ of them evolve oligonephropathy that can carry long-term consequences, such as chronic kidney disease and cardiovascular risks in adulthood [21$23]$. In these kidneys, up to $18 \%$ morphologically abnormal glomeruli are detected that exhibit a dilated Bowman's space and a shrunken glomerular tuft. Also, morphological alterations occur in the organ capsule, and an atypical accumulation of stem cells is registered in the interstitium in the nephrogenic zone $[24,25]$.

Prolongation of nephrogenesis after delivery is a consequent therapeutic approach for affected preterm and low birth weight babies $[26,27]$. However, before this therapeutic process can lead to success, a series of questions need to be answered. For example, whether the process of early delivery, harming metabolites in interstitial fluid or a too early switch off process declining morphogenetic activity in stem cell niches are causing impairment of nephrogenesis has yet to be explored [28-30]. The real problem is that necessary data dealing with the nephrogenic zone and contained stem cell niches of the human fetal kidney are not available. Thus, it is now time to start with a sound analysis of structural, cellular, biological, and physiological details to learn about parenchyma formation during the late phase of gestation. Frequently, it is argued that a wealth of information can be taken over from experiments conducted earlier with transgenic animals [31]. However, most of that data pertains to the organ anlage and initial nephron formation, while substantial structural features and morphogenetic actions in the nephrogenic zone during the last phase of gestation were not a part of earlier biomedical interest.

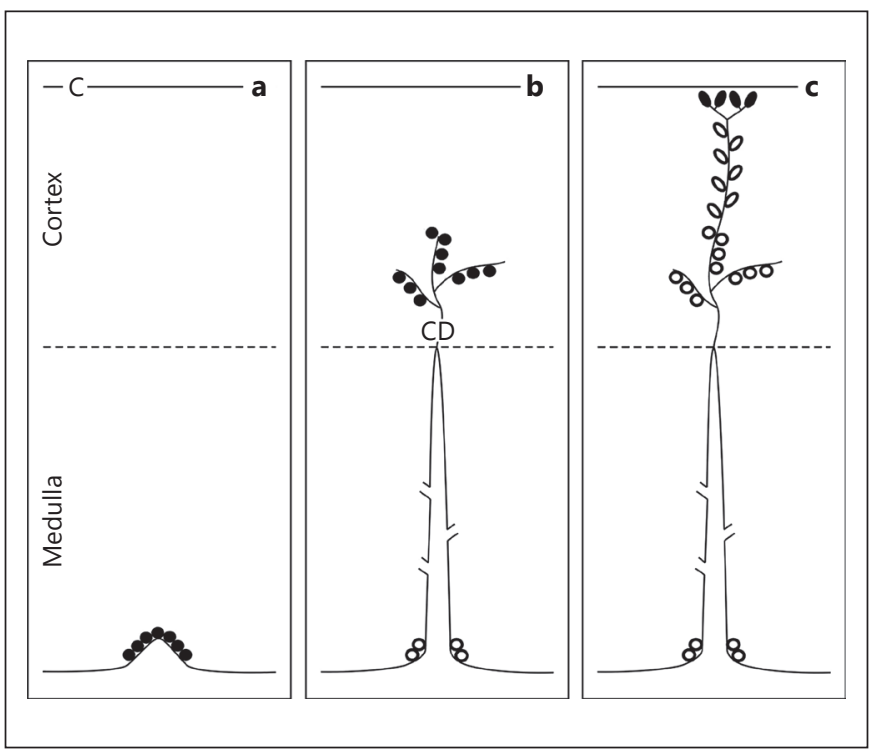

Fig. 1. Schema shows different sites of stem cell niches during human kidney development. a Anlage of the kidney takes place by interaction between the invading ureteric bud and surrounding mesenchyme. First branches of the ureteric bud undergo transformation into the pelvis and calyceal system $[40,41]$. $\mathbf{b}$ There is an average of 8 papillae, while into each an average of 44 ducts of Bellini open. These connect in the cortex elongating collecting duct (CD) tubules that form branches. After the 13th-15th branching in the deep cortex, an elongating CD tubule produces specially arranged CD ampullae, which induce the formation of arcades. c In contrast, the outer cortex branches of a CD tubule elongate towards the capsule $(\mathrm{C})$ to produce laterally situated $\mathrm{CD}$ ampullae. Black - active niche sites; circled - inactive niche sites.

\section{Eccentric Position of the Nephrogenic Zone}

From the organ anlage until birth, development of a mammalian kidney proceeds by an apposition of new parenchyma from the medulla, to the inner, mid, and finally the outer cortex (Fig. 1). This basic developmental process depends on the one hand on epithelial stem cells integrated in the invading ureteric bud. Initiated by branching morphogenesis, it shows a radial and, at the same time, fan-like extension [32-36]. On the other hand, intact development of nephrons principally presupposes successive interactions between branches of the ureteric bud and facing mesenchymal stem cells [37, 38].

The tissue arrangement between the invading ureteric bud and surrounding mesenchyme represents a primary but central niche during organ anlage (Fig. 1a). Regarding the initial development of a human kidney, the first branches of the ureteric bud undergo transformation into 
the pelvis and calyceal systems [39-41]. In contrast to most laboratory animal species, one has to further consider that a human kidney has an average of 8 papillae and in each papilla, there are on average 44 ducts of Bellini open. During the initial development of the cortex, they transmit into the collecting duct (CD) tubules, which elongate towards the capsule to form further branches. Since each of those branches ends blindly and exhibits a dilated form, it was termed CD ampulla $[41,42]$.

Normally, the tip of a CD ampulla and facing mesenchymal cells indicate the site of cell communication, where first the induction and then the initial formation of an individual nephron take place $[43,44]$. However, after the 13th-15th branching has occurred, the start of inner cortex formation is not only at the tip of a CD ampulla but also along the elongating CD tubules (Fig. 1b). As a result, several nephrons can get induced simultaneously, reflecting in turn multiple niche sites [40,41]. During this phase of development, juxtamedullary nephrons including arcades arise. After maturation, each of them exhibits a special morphological pattern as well as a distinct physiological function in the inner cortex of the adult kidney [45-47].

When the formation of arcades is closed, the terminal branches of the 15th generation further elongate as a CD tubule to develop a succession of CD ampullae in the mid and outer cortex. The tip of each strictly points towards the capsule. It is often considered that their shape is not different from those that develop during the earlier phase of branches in the inner cortex. During the formation of the mid and outer cortex, they are less ballooned, more pointed and sometimes triangular [40,41]. During ongoing radial extension of parenchyma and at the end of gestation, each branch of a CD tubule continues to elongate towards the organ capsule leaving the $\mathrm{CD}$ ampullae to induce nephrons on each tip in a more or less geometric pattern [44] (Fig. 1c). According to previously published literature, these branches are clearly lateral (monopodial) branches and have been termed the open divided system [40]. Finally, at the 38th week of gestation, the bifid branching of the CD tubule including formation of CD ampullae is ended by an unknown molecular mechanism [40].

\section{View by Optical Microscopy}

The nephrogenic zone of a fetal human kidney extends as a narrow band along the entire inner side of the organ capsule. To prevent damage during isolation, one should best hold a fetal kidney only on its hilum and should not touch the capsule with fine forceps. To illustrate the nephrogenic zone, a human kidney of gestational age between weeks 16 and 18 was selected from the stock of preparations used for the Course of Microscopic Anatomy for Medical Students at the University of Regensburg. According to routine methods, specimens were fixed in paraformaldehyde solution and embedded in paraffin wax. Then sections of $5 \mu \mathrm{m}$ thickness were made and stained by hematoxylin-eosin solution for analysis by optical microscopy. Images were taken with a digital camera and processed with CorelDRAW X7 (Corel Corporation, Munich, Germany). To obtain information about morphological coordinates, readings were performed with the same program.

Histological sections cut incidentally for microscopic analysis do not help to obtain comparable views of the nephrogenic zone for evaluation. To obtain targeted but comparable perspectives, selected blocks of parenchyma must be orientated before embedding and during histological cutting. Best views are possible when the section plane lies parallel to the lumen of CD tubules and exactly perpendicular to the organ capsule (Fig. 2). When this kind of orientation is applied, the nephrogenic zone including the aligned stem cell niches are observed in a vertical view. It lies in the inner side of the organ capsule and spans a width of $60-100 \mu \mathrm{m}$ depending on the species [48-50]. Obviously due to metabolic peculiarities, the nephrogenic zone exhibits a slightly pronounced coloration in sections stained by hematoxylin-eosin solution. For this reason, it is also called the "blue strip" [25, 27].

Prominent structures of the nephrogenic zone are the endings of $\mathrm{CD}$ tubules. Depending on section planes, one can see branching sites connected with ureteric bud-derived $C D$ ampullae (Fig. 2). Their tips are facing metanephric mesenchyme. Superimposed on both structures is the organ capsule. When nephrogenesis is active, the initial formation of nephrons in the form of aggregated cells in mesenchyme (pretubular aggregate), polarized renal vesicles or comma-shaped bodies are visible at the upper lateral aspect of $\mathrm{CD}$ ampullae. Overall, the contained components represent the cell biological setting to maintain stemness, branching morphogenesis, induction, and initial formation of individual nephrons. In this context, one must consider that all of the different tasks are mastered by a set of morphogens and nephron progenitor cells $[51,52]$.

In the late fetal phase of kidney development, only bifid branches of a CD tubule determine the radial exten- 


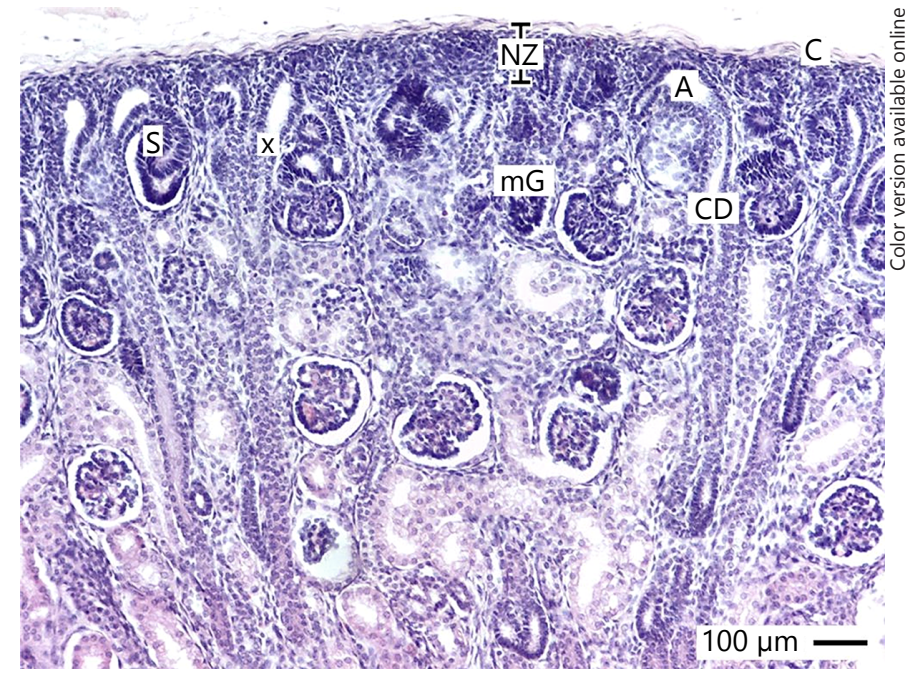

Fig. 2. Optical microscopy shows the nephrogenic zone (NZ) in the outer cortex of a human fetal kidney. The histological section lies perpendicular to the organ capsule $(\mathrm{C})$ and parallel to the lumen of collecting duct tubules (CD). Beyond the capsule branches are seen, which exhibit a neck (X) and a CD ampulla (A). Nephrogenic mesenchymal stem cells appear between the tip of a CD ampulla and the inner side of the capsule. At the upper lateral aspect of a CD ampulla, pretubular aggregates or renal vesicles indicate active nephrogenesis. At its lower lateral aspect, S-shaped bodies (S) show the initial development of a glomerulus and tubulogenesis. $\mathrm{mG}$, maturing glomerulus.

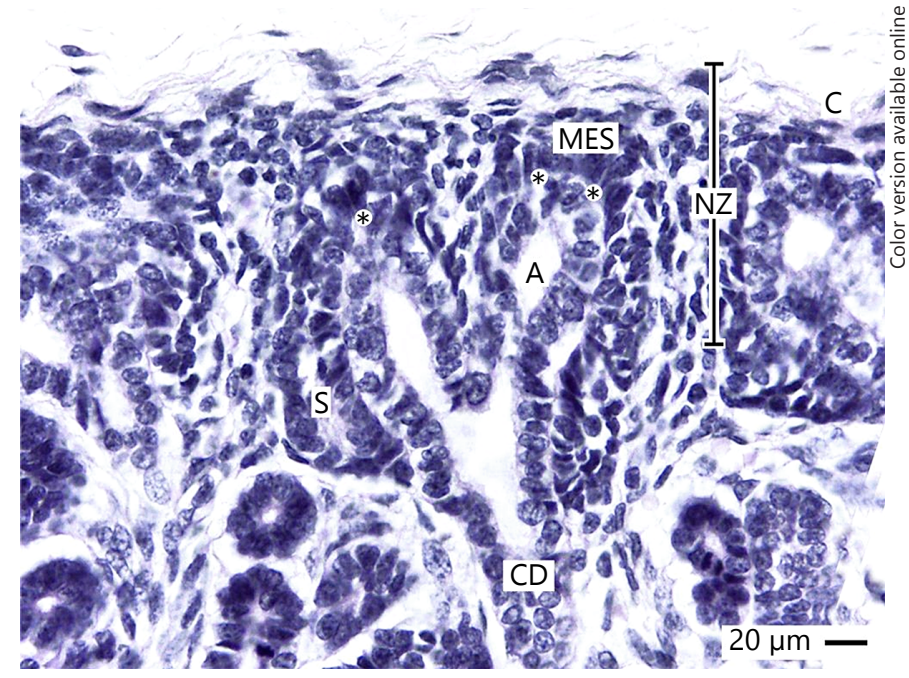

Fig. 3. Optical microscopy depicts in the nephrogenic zone (NZ) of a fetal human kidney a stem cell niche. By branching of the collecting duct (CD) tubule a CD ampulla (A) arise. Epithelial stem cells contained in the tip of a CD ampulla face 2 layers of mesenchymal stem cells (MES). In the center of the niche, an interface (small asterisks) separates epithelial and mesenchymal stem cell bodies. S, shaped body. sion of parenchyma in the outer cortex (Fig. 1c) [40]. As seen in a related micrograph, a branch points towards the capsule to form a CD ampulla at its end (Fig. 3). The epithelial stem cells contained here exhibit a simple cuboidal shape. In the optical microscope, one can see that the tip of a CD ampulla does not touch the neighboring mesenchymal stem cells, but is separated by a striking interface as was observed in neonatal rabbit kidney [53]. On sections oriented along the axis of lining $\mathrm{CD}$ tubules, one can also see that only 2 layers of mesenchymal stem cells occur between the tip of a CD ampulla and the inner side of capsule. Most important in this special perspective: the formation of a so-called cap mesenchyme is not visible. Occasionally, a multilayered cap mesenchyme becomes visible, when sections are analyzed that lie oblique to the axis of related CD tubules. Finally, the interface between the mesenchymal stem cell layer $\left(\mathrm{GDNF}^{+} / \mathrm{Six}^{+} / \mathrm{CIT}\right.$ $\left.\mathrm{ED}^{+}\right)$juxtaposed to the tip of a $\mathrm{CD}$ ampulla containing epithelial stem cells defines the center of an individual stem cell niche enabling induction and initial formation of an individual nephron [51, 52, 54-56].

\section{Metric Parameters}

To obtain the first information about metric data of the human nephrogenic zone (Fig. 2, 3), the diameter of a $\mathrm{CD}$ tubule near the branching site, distance between the tips of CD ampullae, length of a CD ampulla, and its diameter were determined (Table 1; Fig. 4). Moreover, the distance between the tip of a CD ampulla and the inner side of the organ capsule (Fig. 4), the thickness of the mesenchymal cell layers (Fig. 4), and the distance between the branching site of a CD ampulla and the capsule (Fig. 4) were determined. Finally, the distance between the lateral aspects of CD ampullae (Fig. 4) was stated.

Without any claim of completeness and as a preliminary result, Table 1 shows the coordinates of the nephrogenic zone recorded by morphometry. For example, it was detected that the diameter of a CD tubule is between 20 and $42 \mu \mathrm{m}$ (mean $28 \mu \mathrm{m}, n=10$ ). The distance between the tip of a CD ampulla to the next is between 107 and 211 $\mu \mathrm{m}$ (mean $131 \mu \mathrm{m}, n=10$ ). When the CD tubule shows branching, the limb lines into a CD ampulla. Its length is between 78 and $116 \mu \mathrm{m}$ (mean $81 \mu \mathrm{m}, n=10$ ), while its diameter is between 47 and $86 \mu \mathrm{m}$ (mean $68 \mu \mathrm{m}, n=10$ ). Between the tip of a CD ampulla and the inner side of the organ capsule, a distance ranging between 16 and $41 \mu \mathrm{m}$ (mean $32 \mu \mathrm{m}, n=10$ ) was found. The thickness of the recognized mesenchymal cell layers localized between the 
Table 1. Monitoring sites to determine coordinates of the nephrogenic zone and contained stem cell niches in a human fetal kidney

\begin{tabular}{|c|c|c|c|c|c|}
\hline 1 & Diameter of CD tubule & 20 & 42 & 28 & 10 \\
\hline 2 & Distance between CD ampulla tips & 107 & 211 & 131 & 10 \\
\hline 3 & Length of CD ampulla & 78 & 116 & 81 & 10 \\
\hline 5 & Distance between the tip of CD ampulla and inner side of organ capsule & 16 & 41 & 32 & 10 \\
\hline 6 & Thickness of mesenchymal cell layers & 12 & 29 & 22 & 10 \\
\hline 7 & Distance between the branching site of CD ampulla and inner side of capsule & 49 & 129 & 83 & 10 \\
\hline 8 & Distance between lateral aspects of CD ampullae & 34 & 106 & 78 & 10 \\
\hline
\end{tabular}

inner side of the capsule and the tip of a CD ampulla is between 12 and $29 \mu \mathrm{m}$ (mean $22 \mu \mathrm{m}, n=10)$. The distance between the branching site of a CD ampulla and the inner side of the capsule is between 49 and $129 \mu \mathrm{m}$ (mean 83 $\mu \mathrm{m}, n=10$ ). Lateral aspects of neighboring CD ampullae show a distance between 34 and $106 \mu \mathrm{m}$ (mean $78 \mu \mathrm{m}, n=$ $10)$.

\section{Constant and Variable Patterns}

Optical microscopy (Fig. 2, 3) as well as data given in Table 1 revealed that in the nephrogenic zone of fetal kidney relatively constant but also rather variable parameters occur. First, stem cell niches consisting of the tip of a CD ampulla and juxtaposed mesenchymal stem cells are not randomly distributed but are constantly and closely arranged in the capsule. The distance between the tip of a $\mathrm{CD}$ ampulla and the inner side of the organ capsule is on average $32 \mu \mathrm{m}$ (Fig. 4; Table 1). Surprisingly, only 2 layers of mesenchymal stem cells occur between the tip of a CD ampulla and the inner side of the organ capsule. Both mesenchymal cell layers have a mean thickness of $22 \mu \mathrm{m}$ (Fig. 4; Table 1). The distance between the branching site of a CD ampulla and the inner side of the capsule is on average $83 \mu \mathrm{m}$ (Fig. 4; Table 1). Moreover, a CD ampulla has $81 \mu \mathrm{m}$ length on average (Fig. 4; Table 1) and a diameter of $68 \mu \mathrm{m}$ (Fig. 4; Table 1).

Not only constant parameters were recorded, but the data also exhibit that the distance from a CD ampulla tip to the next varies, and this can be between 107 and 211 resulting in a mean of $131 \mu \mathrm{m}$ (Fig. 4; Table 1). The space

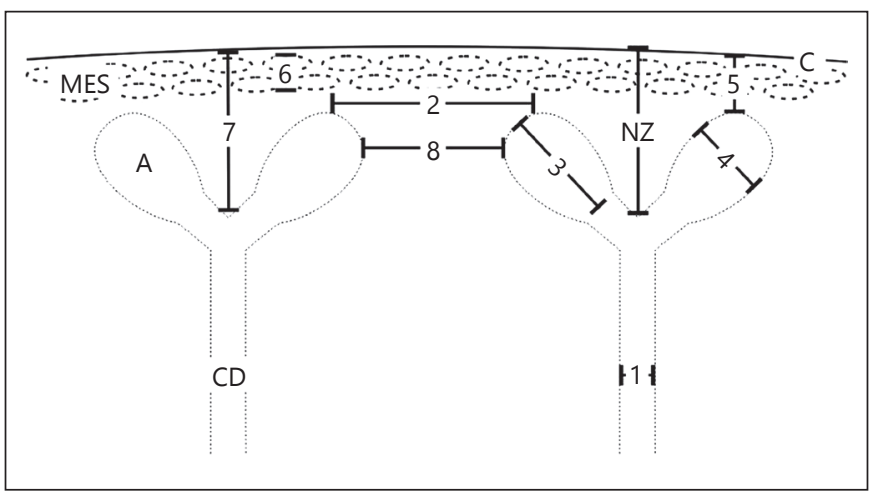

Fig. 4. Schematic illustration informs about monitoring sites to determine coordinates of the nephrogenic zone (NZ) in a human fetal kidney: Diameter of CD tubule near the branching site (1), distance between CD ampulla (A) tips (2), length (3), and diameter of a CD ampulla (4). In addition, the distance between the tip of a $\mathrm{CD}$ ampulla and the inner side of the organ capsule $(\mathrm{C} ; 5)$, thickness of the mesenchymal cell (MES) layers (6), distance between the branching site of a CD ampulla and the capsule (7) and the distance between lateral aspects of CD ampullae (8) were determined.

between lateral aspects of a CD ampulla to the next is between 34 and 106, resulting in an average of $78 \mu \mathrm{m}$ (Fig. 4; Table 1).

\section{Model True to Scale}

The recordings shown in Table 1 made it possible to process them and to create a scaled schema of the human renal stem cell niche for the first time (Fig. 5a). In this at- 


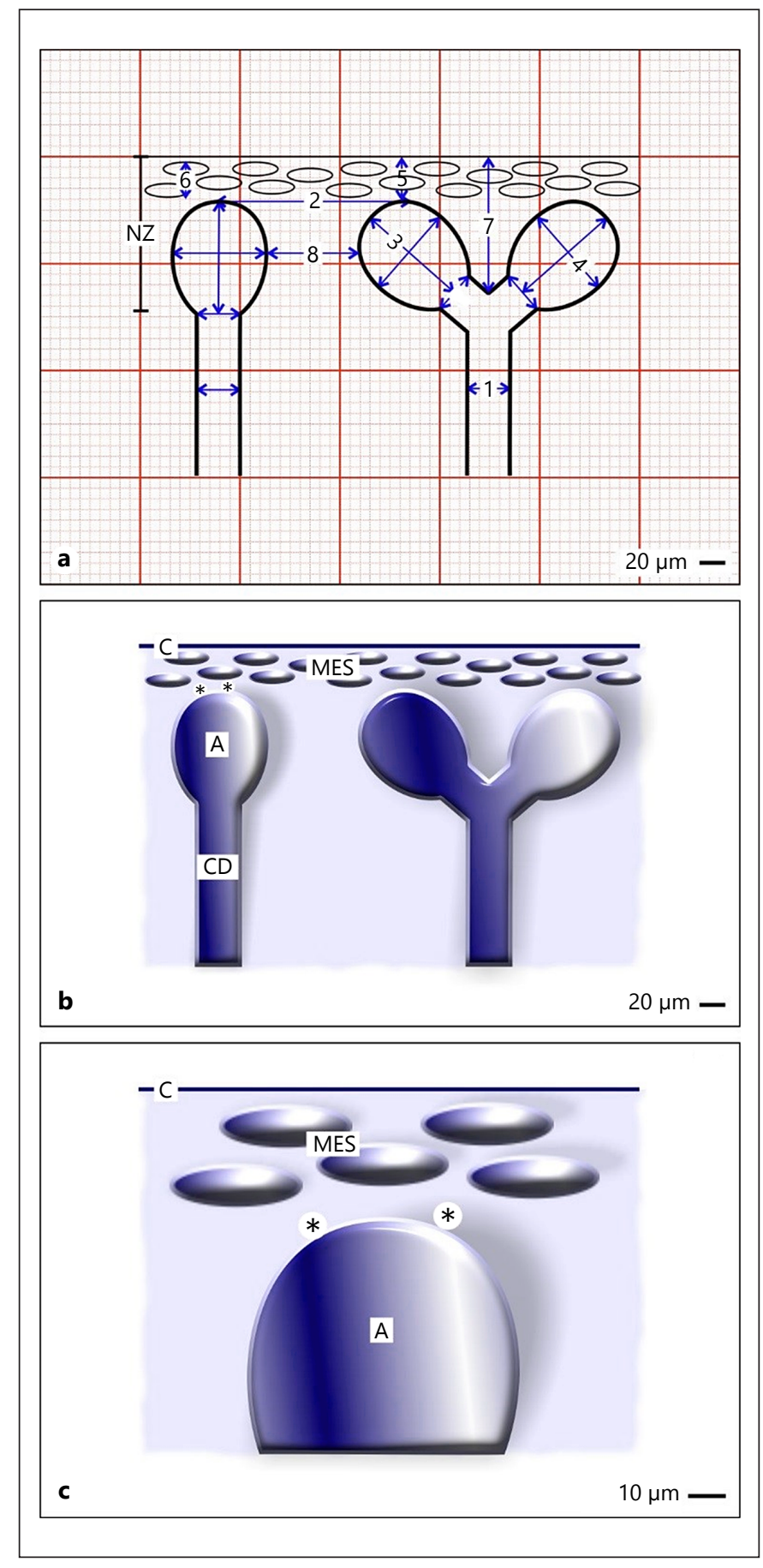

Fig. 5. Processing of a stem cell niche in scale of the human fetal kidney according to data shown in Table 1 . a, b Frontal view to a niche is on the right, while its lateral aspect is demonstrated on the left side. a Spatial orientation of a collecting duct (CD) tubule, CD ampulla (A), nephrogenic mesenchyme (MES), and the organ capsule $(\mathrm{C})$ is shown on graph. $\mathbf{b}$ In a next step, illustration made on graph is transformed to a pseudo 3D illustration. c Schema shows in higher magnification a niche in scale. Beyond the capsule, the tip of a CD ampulla and mesenchymal stem cells are visible that are separated by an interface (asterisk). tempt, only compounds needed for nephron induction such as the end of a CD tubule including its branching site, position of a CD ampulla, both layers of nephrogenic mesenchyme cells, and the covering capsule were visualized (Fig. 5b, c). Later and for an extended version, aggregation sites of mesenchyme, exact position of mesenchymal to epithelial transition, polarized renal vesicles, and S-shaped bodies may be supplemented. In a further version, it becomes possible to add forming vessels at the branching CD tubule and sprouting endothelial cells at the lateral aspect of a CD ampulla and on the lower cleft of S-shaped bodies, where the glomerular tuft arises. Later, when exact immunohistochemical and ultrastructural data are available, the position of cell layers within the capsule and distribution of neighboring interstitial cells can be included. Moreover, when data from pathological findings of preterm and low birth weight babies that died due to different reasons are completed, patterns become visible, which help to determine the destructive structural processes. Finally, introduced and envisaged data make it possible to produce a realistic model, true to scale for example, by a 3D printer.

\section{Layout between Niche and Capsule}

For a long time, it was assumed that niches integrated in the nephrogenic zone operate randomly and in an unstructured area. However, the preliminary data signals constant and variable patterns (Table 1). Viewing the niches within the nephrogenic zone with an optical microscope shows a constant spatial proximity to the inner side of the capsule (Fig. 2, 3; Table 1). In contrast, the lateral distance of niches varies greatly (Table 1 ). The actual problem is that there is no ultrastructural data available for the human fetal kidney. However, for neonatal rabbit kidneys, it was shown that the area between the organ capsule, mesenchyme, and the tip of a CD ampulla exhibits a surprisingly constant layout.

Transmission electron microscopy showed flat fibroblasts and collagen fiber bundles not labeled by tannic acid at the outer side of the organ capsule in zone 1 [57]. In zone 2, collagen fiber bundles appear that are intensively labeled by tannic acid. In zone 3 , cells with numerous interdigitating protrusions occur. Astonishingly and rarely noticed, at this site intra- and extracellular tunnels are formed, which seem to provide the niche area with nutrition and respiratory gas. A basal lamina covers part of the cells that occur here so that they correspond to smooth muscle cells. In zone 4, only 2 layers of mesenchy- 
mal stem cells are present. Due to their close vicinity to the tip of a CD ampulla, the inner mesenchymal cell layer corresponds to $\mathrm{GDNF}^{+} / \mathrm{Six}^{+} / \mathrm{CITED} 1^{+}$cells $[51,52]$. It is particularly important that these cells send out projections to establish functional contacts with neighboring epithelial cells via tunneling nanotubes [58]. Indicated as zone 5, a striking interface separates the mesenchymal from epithelial stem cell bodies in the center of a niche.

\section{Mechanical Fastening of Niches}

What is remarkable is that during kidney growth, including radial extension of parenchyma, the nephrogenic zone and contained niches are in close contact with the organ capsule (Fig. 1c, 2, 3). For the human kidney, there is no information available, but in the neonatal rabbit kidney it is due to microfibers linking each of the niches with the capsule [57]. They originate on the basal lamina covering the epithelial stem cells at the tip of a CD ampulla. Microfibers positively labeled by anti-collagen types I, II, III, and IV or Soybean agglutinin span through both layers of mesenchymal stem cells to end on the inner side of the capsule. Thus, occurrence of microfibers within the renal niche depicts that epithelial and mesenchymal stem cells do not meet by accident but are part of a meshed mechanical mount.

\section{Fine Positioning of Stem Cells}

Encountering of cells within an individual niche appears to be adjusted by the secreted bone morphogenetic protein antagonist Cerberus homolog1, Ret proto oncogene, and ETS translocation variant $4[59,60]$. The molecular motion triggers the tip of a CD ampulla in precise position to the nearest cells of mesenchyme. The actual time lapse imaging shows that the mesenchymal cells are motile and they attach and detach from the CD ampulla tip across time [61]. While approaching, these cells must acquire competence so that they can respond to morphogens. So far it is known, the special operation is under the control of protocadherin (cadherin family member14: FAT4/dachsous cadherin related1: Dchs1) signaling [62]. As a result, epithelial stem cells in the tip of a CD ampulla meet $\mathrm{GDNF}^{+} / \mathrm{Six} 2^{+} / \mathrm{CITED}^{+}$mesenchymal cells $[51,52]$. At the same time, a reciprocal exchange of morphogens within the niche takes place [37, 38]. When the signaling for the process of induction is successful, a few mesenchymal cells separate, aggregate,

Nephrogenic Zone in Human Fetal Kidney and perform a mesenchyme-to-epithelial transition (MET) to develop into a renal vesicle at the lateral aspect of the related CD ampulla [63]. For remaining and not induced mesenchymal cells, the transcription factor Zeb1 controls the balance between further proliferation, migration, and apoptosis [64]. Thus, repeated branching of a CD tubule, approximation of a CD ampulla tip to competent mesenchymal cells, and finally timely induction superimposes new nephrons beyond the capsule [42].

\section{Challenge for Young Scientists}

Numerous micrographs and free hand design illustrations are available of different animal species dealing with branching of the ureteric bud, surrounding mesenchyme and initial nephron formation. In contrast, surprisingly few and mostly incomplete illustrations exist, which show the nephrogenic zone and contained stem cell niches in human fetal kidney. It is also surprising that relevant investigations made by scanning or transmission electron microscopy do not exist. However, for teaching medical or graduate students in microscopic anatomy and when holding training courses for young pediatricians, pathologists, pharmacologists, and scientists working in the biomedical research field, solid knowledge in microanatomy and ultrastructure of the nephrogenic zone in human fetal kidney is indispensable.

To introduce the complex area in a first attempt, prominent niche sites during human kidney development were schematically illustrated (Fig. 1). During the organ anlage, one can define the primary niche as a centered juxtaposition of the invading ureteric bud and surrounding mesenchyme (Fig. 1a). When anlage of the presumptive medulla is finished, the development of parenchyma starts in the inner cortex, showing ureteric bud derived branches along elongating CD tubules including focal points, which represent special but less considered niche sites for the development of arcades (Fig. 1b) [40, $41,45-47]$. Then, at the end of gestation, according to the position of $\mathrm{CD}$ ampullae, all of the active niches are aligned within the nephrogenic zone in close quarters with the organ capsule (Fig. 1c, 2, 3). A look at the illustrations presented here demonstrates that the number of stem cells in the primary niche during organ anlage (Fig. 1a) and inner cortex formation (Fig. 1b) is unexpectedly small when compared to countless niches in the nephrogenic zone during the late phase of gestation (Fig. 1c, 2). The increased number of stem cell niches, 
their unique spatial distribution beyond the capsule, and their distinct but terminated tasks at birth raise valid questions that need to be answered.

\section{Call for Future Investigations}

Regarding the nephrogenic zone and aligned stem cell niches (Fig. 2, 3), one question that remains unanswered is whether morphogenetic activity of a single niche is controlled by a site-specific but intrinsic mechanism or whether a master system supervises all the niches in parallel. It seems that morphogenetic switching off of all the niches simultaneously shortly before birth speaks for the control by an effective master system. Furthermore, there might be differences in morphogenetic signaling, when kidney anlage is being established (Fig. 1a), development of medulla takes place, and arcades arise in the inner cortex (Fig. 1b). A further switching over of the developmental program results in the development of a new kind of $\mathrm{CD}$ ampullae, stimulating in turn the induction of a new nephron type in the mid and then in the outer cortex until birth (Fig. 1c) [40, 41].

Moreover, it has been known for years that morphogenetic interactions between epithelial stem cells in the tip of a CD ampulla and juxtaposed nephrogenic mesenchymal $\left(\mathrm{GDNF}^{+} / \mathrm{Six}^{+} / \mathrm{CITED}^{+}\right)$cells lead to induction and initial formation of a nephron [37, 38, 51, 52]. It is obvious that the micrographs and morphometric data shown here are still sparse, that further parameters must be considered and the stock of measurements must be expanded (Table 1). Nevertheless, some striking insights were made possible. For example, to date it is hardly perceived that during the late phase of parenchyma formation, induction of nephrons occurs in close vicinity, in a structured pattern and in a special microenvironment of extracellular matrix on the inner side of the organ capsule (Table 1; Fig. 2, 3) [53, 57, 58]. Besides an intact morphogenetic activity, the site, amount, and arrangement of competent cells within niches must also be carefully analyzed when causes of impaired nephrogenesis in preterm and low birth weight babies are under debate $[20,23]$.

Although not for current routine diagnosis, an elaboration of whether the previously mentioned morphological parameters are different in preterm or low birth weight babies that died of various causes is necessary for detailed pathologic research in the near future $[27,50]$. Reading those case-specific coordinates according to the schema introduced here (Table 1; Fig. 5b, c), in combination with modern computer-assisted techniques such as laser scanning immunohistochemistry or optical projection tomography could help to more precisely separate an inactive from active status of the nephrogenic zone and integrated niches [65-67].

Finally, knowledge about microanatomy of the nephrogenic zone is a prerequisite, when local drug delivery prolonging nephrogenesis in preterm infants is in the focus of an innovative therapeutic approach [30]. Although it is not often taken into consideration, one must bear in mind that the nephrogenic zone has an incomplete vascular supply that makes controlled drug delivery difficult [68]. Consequently, it is necessary to first find a suitable path for drug application via infusion or local stem cell implantation. In this connection, it is obligatory to observe not only the necessary morphogenetic impulses, but also to have a close look at the local microenvironment and the morphogen actions, in which a stem or progenitor cell, respectively a niche, is biologically active $[69,70]$. While it is obvious that the creation of the schema introduced here cannot answer the questions raised, it gives the first detailed information about the complex microanatomy of the nephrogenic zone and contained stem cell niches. For these reasons, the present work is an invitation, especially for young people, to re-investigate the nephrogenic zone and to complete the temporary data presented here with comprehensive knowledge and adequate techniques as it has recently been requested [71].

\section{Acknowledgements}

This project was supported by Emeriti Research Fund of the University of Regensburg, DE-93053 Regensburg, Germany.

\section{Disclosure Statement}

The author has no conflicts of interest to declare.

References

1 Little MH, Kairath P: Regenerative medicine in kidney disease. Kidney Int 2016;90:289299.

2 Meyer-Schwesinger C: The role of renal progenitors in renal regeneration. Nephron 2016; 132:101-109.

3 Aggarwal S, Moggio A, Bussolati B: Concise review: stem/progenitor cells for renal tissue repair: current knowledge and perspectives. Stem Cells Transl Med 2013;2:1011-1019.

4 Herrera M, Miotsou M: Stem cells: potential and challenges for kidney repair. Am J Physiol Renal Physiol 2014;306:F12-F23. 
5 Lam AQ, Bonventre JV: Regenerating the nephron with human pluripotent stem cells. Curr Opin Organ Transplant 2015;20:187192.

6 Peired AJ, Sisti A, Romagnani P: Mesenchymal stem cell based therapy for kidney disease: a review of clinical evidence. Stem Cells Int 2016;4798639.

7 Morigi M, Benigni A: Mesenchymal stem cells and kidney repair. Nephrol Dial Transplant 2013;28:788-793.

8 Bussolati B, Camussi G: Therapeutic use of human renal progenitor cells for kidney regeneration. Nat Rev Nephrol 2015;11:695706.

9 Burst V, Pütsch F, Kubacki T, Völker LA, Bartram MP, Müller Ru, Gillis M, Kurschat CE, Grundmann F, Müller-Ehmsen J, Benzing T, Teschner S: Survival and distribution of injected haematopoietic stem cells in acute kidney injury. Nephrol Dial Transplant 2013;28: 1131-1139.

10 Katari R, Eggar L, Wong T, Boey A, Mancone S, Igel D, Callese T, Voigt M, Tamburini R, Zambon JP, Perin L, Orlando G: Tissue-engineering approaches to restore kidney function. Curr Diab Rep 2015;15:69.

11 Bonandrini B, Figliuzzi M, Papadimou E, Morigi M, Perico N, Casiraghi F, Dipl C, Sangalli F, Corti S, Benigni A, Remuzzi A, Remuzzi G: Recellularization of well-preserved acellular kidney scaffold using embryonic stem cells. Tissue Engineering Part A 2014;20: 1486-1498.

12 Little MH, Combes AN, Takasato M: Understanding kidney morphogenesis to guide renal tissue regeneration. Nat Rev Nephrol 2016;12:624-635.

13 Cho SH, Noh JR, Cho MY, Go MJ, Kim YH, Kang ES, Kim YH, Lee CH, Lim YT: An injectable collagen/poly $(\gamma$-glutamic acid $)$ hydrogel as a scaffold of stem cells and a-lipoic acid for enhanced protection against renal dysfunction. Biomater Sci 2017;5:285-294.

14 Rinkevich Y, Montoro DT, Contreras-Trujillo H, Harari-Sternberg O, Newman AM, Tsai JM, Lim X, van Amerongen R, Bowman A, Januszyk $M$, et al: In vivo clonal analysis reveals lineage-restricted progenitor characteristics in mammalian kidney development, maintenance, and regeneration. Cell Rep 2014;7:1270-1283.

15 Sallustio F, Serino G, Schena FP: Potential reparative role of resident adult renal stem/progenitor cells in acute kidney injury. Biores Open Access 2015;4:326-333.

16 Hishikawa K, Takase O, Yoshikawa M, Tsujimura T, Nangaku M, Takato T: Adult stemlike cells in kidney. World J Stem Cells 2015; 7:490-494.

17 Kumar S, Liu J, Pang P, Krautzberger AM, Reginensi A, Akiyama H, Schedl A, Humphreys BD, McMahon AP: Sox9 activation highlights a cellular pathway of renal repair in the acutely injured mammalian kidney. Cell Rep 2015;12:1325-1338.
18 Dziedzic K, Pleniceanu O, Dekel B: Kidney stem cells in development, regeneration and cancer. Semin Cell Dev Biol 2014;36:57-65.

19 Mari C, Winyard P: Concise review: understanding the renal progenitor cell niche in vivo to recapitulate nephrogenesis in vitro. Stem Cells Transl Med 2015;4:1463-1471.

20 Stoops C, Sims B, Griffin R, Askenazi DJ: Neonatal acute kidney injury and the risk of intraventricular hemorrhage in the very low birth weight infant. Neonatology 2016;110: 307-312.

21 Gubhaju L, Sutherland MR, Yoder BA, Zulli A, Bertram JF, Black MJ: Is nephrogenesis affected by preterm birth? Studies in a non-human primate model. A J Physiol Renal Physiol 2009;297:F1668-F1677.

22 Saint-Faust M, Boubred F, Simeoni U: Renal development and neonatal adaption. Am J Perinatol 2014;31:773-780.

23 Abitbol CL, DeFreitas MJ, Strauss J: Assessment of kidney function in preterm infants: lifelong implications. Pediatr Nephrol 2016; 31:2213-2222.

24 Fanni D, Gerosa C, Nemolato S, Mocci C, Pichiri G, Coni P, Congiu T, Piludu M, Piras M, Fraschini M, Zaffanello M, Iacovidou N, Van Eyken P, Monga G, Faa G, Fanos V: "Physiological" renal regenerating medicine in VLBW preterm infants: could a dream come true? J Matern Fetal Neonatal Med 2012;3: $41-48$.

25 Fanni D, Sanna A, Gerosa C, Puddu M, Faa G, Fanos V: Each niche has an actor: multiple stem cell niches in the preterm kidney. Ital J Pediatr 2015;41:78.

26 Kandasamy Y, Smith R, Wright IM, Lumbers ER: Extra-uterine renal growth in preterm infants: oligonephropathy and prematurity. $\mathrm{Pe}-$ diatr Nephrol 2013;28:1791-1796.

27 Fanos V, Castagnola M, Faa G: Prolonging nephrogenesis in preterm infants: a new approach for prevention of kidney disease in adulthood? Iran J Kidney Dis 2015;9:180-185.

28 Mussap M, Ferrari M, Fanos V: Laboratory medicine meets precision medicine: The paradigm of metabolomics in perinatology: proposals from the 4th international conference on neonatal and pediatric laboratory medicine. Clin Chem Acta 2015;451(pt A):1-3.

29 Noto A, Fanos V, Dessi A: Metabolomics in newborns. Adv Clin Chim 2016;74:35-61

30 Minuth WW: Action plan for prolongation of nephrogenesis in preterm and growth restricted babies: Explore ultrastructure of the nephrogenic zone, identify a molecular target, select a viable drug and find a path for administration. Drug Res 2017, DOI: 10.1055/s-0043-113637.

31 Potter SS, Brunskill EW, Patterson LT: Defining the genetic blueprint of kidney development. Pediatr Nephrol 2011;26:1469-1478.

32 Meyer TN, Schwesinger C, Bush KT, Stuart RO, Rose DW, Shah MM, Vaughn DA, Steer DL, Nigam SK: Spatiotemporal regulation of morphogenetic molecules during in vitro branching of the isolated ureteric bud: toward a model of branching through budding in the developing kidney. Dev Biol 2004;275:44-67.

33 Chi X, Michos O, Shakya R, Riccio P, Enomoto H, Licht JD, Asai N, Takahashi M, Ohgami N, Kato M, et al: Ret-dependent cell rearrangements in the Wolffian duct epithelium initiate ureteric bud morphogenesis. Dev Cell 2009;17:199-209.

34 Costantini F: Genetic controls and cellular behaviors in branching morphogenesis of the renal collecting duct system. Wiley Interdiscip Rev Dev Biol 2012;1:693-713.

35 Short KM, Combes AN, Lefevre J, Ju AL, Georgas KM, Lamberton T, Cairncross O, Rumballe BA, McMahon AP, Hamilton NA, et al: Global quantification of tissue dynamics in the developing mouse kidney. Dev Cell 2014;29:188-202.

36 Short KM, Smyth IM: The contribution of branching morphogenesis to kidney development and disease. Nat Rev Nephrol 2016;12: 754-767.

37 Carroll TJ, Das A: Defining the signals that constitute the nephron progenitor niche. J Am Soc Nephrol 2013;24:873-876.

38 Krause M, Rak-Raszewska A, Pietilä I, Quaggin SE, Vainio S: Signaling during kidney development. Cells 2015;4:112-132.

39 Osathanondh V, Potter EL: Development of human kidney as shown by microdissection. II. Renal pelvis, Calyces, and papillae. Arch Pathol 1963;76:277-289.

40 Oliver J: Nephrons and Kidneys. New York, Hoeber Medical Division, Harper \& Row, 1968.

41 Al-Awqati O, Goldberg MR: Architectural patterens in branching morphogenesis in the kidney. Kidney Int 1998;54:1832-1842.

42 Blake J, Rosenblum ND: Renal branching morphogenesis: morphogenetic and signaling mechanisms. Semin Cell Dev Biol 2014; 36:2-12.

43 Potter EL: Development of the human glomerulus. Arch Pathol. 1965;80:241-55.

44 Osathanondh V, Potter EL: Development of human kidney as shown by microdissection. IV. Development of tubular portions of nephrons. Arch Pathol 1966;82:391-402.

45 Göransson A, Sjöquist M, Ulfendahl HR: Superficial and juxtamedullary nephron function during converting enzyme inhibition. Am J Physiol 1986;251:F25-F33.

46 Kishore BK, Mandon B, Oza NB, DiGiovanni SR, Coleman RA, Ostrowski NL, Wade JB, Knepper MA: Rat renal arcade segment expresses vasopressin-regulated water channel and vasopressin V2 receptor. J Clin Invest 1996;97:2763-2771.

47 Beierwalters WH, Harrison-Bernhard LM, Sullivan JC, Mattson DL: Assessment of renal function; clearance, the renal microcirculation, renal blood flow, and metabolic balance. Compr Physiol 2013;3:165-200.

48 Fanos V, Loddo C, Puddu M, Gerosa C, Fanni D, Ottonello G, Faa G: From ureteric bud to the first glomeruli: gens, mediators, kidney alterations. Int Urol Nephrol 2015;47:109-116. 
49 Rumballe BA, Georgas KM, Combes AN, Ju AL, Gilbert T, Little MH: Nephron formation adopts a novel spatial topology at cessation of nephrogenesis. Dev Biol 2011;360:110-122.

50 Faa G, Sanna A, Gerosa C, Fanni D, Puddu M, Ottonello G, Van Eyken P, Fanos V: Renal physiological regenerative medicine to prevent chronic renal failure: should we start at birth? Clin Chem Acta 2015;444:156-162.

51 Combes AN, Davies JA, Little MH: Cell-cell interactions driving kidney morphogenesis. Curr Top Dev Biol 2015;112:467-508.

52 Da Sacco S, Thornton ME, Petrosyan A, Lavarreda-Pearce $M$, Sedrakyan S, Grubbs BH, De Filippo RE, Rerin L: Direct isolation and characterization of human nephron progenitors. Stem Cell Transl Med 2016;6:419-433.

53 Minuth WW, Denk L: Cell projections and extracellular matrix cross the interstitial interface within the renal stem/progenitor cell niche: accidental, structural or functional cues? Nephron Exp Nephrol 2012;122:131140.

54 Piludu M, Fanos V, Congiu T, Piras M, Gerosa C, Mocci c, Fanni D, Nemolato S, Muntoni S, Iacovidou N, Faa G: The pine-cone body: an intermediate structure between the cap mesenchyme and the renal vesicle in the developing nod mouse kidney revealed by an ultrastructural study. J Matern Fetal Neonatal Med 2012;25:41-48.

55 Brown AC, Muthukrishnan SD, Guay JA, Adams DC, Schafer DA, Fetting JL, Oxburgh L: Role of compartmentalization in nephron progenitor differentiation. Proc Natl Acad Sci U S A 2013;110:4641-4645.

56 O’Brien LL, Guo Q, Lee YJ, Tran T, Benazet JD, Whitney PH, Valouev A, McMahon AP: Differential regulation of mouse and human nephron progenitors by the six family of tran- scriptional regulators. Development 2016; 143:595-608.

57 Minuth WW, Denk L: Structural links between the renal stem/progenitor cell niche and the organ capsule. Histochem Cell Biol 2014;141:458-471.

58 Minuth WW, Denk L: When morphogenetic proteins encounter special extracellular matrix and cell-cell connections at the interface of the renal stem/progenitor cell niche. Anat Cell Biol 2015;48:1-9.

59 Riccio P, Cebrian C, Zong H, Hippenmeyer S, Constantini F: Ret and Etv4 promote directed movements of progenitor cells during renal branching morphogenesis. PLoS Biol 2016; 14:e1002382

60 Chi L, Saarela U, Railo A, PrunskaiteHyyryläinen R, Skovorodkin I, Anthony S, Katsu K, Liu Y, Shan J, Salgueiro AM, Belo JA, Davies J, Yokouchi Y, Vainio SJ: A secreted BMP antagonist, CER1, fine tunes the spatial organization of the ureteric bud tree during mouse kidney development. PLoS One 2011;6:e27676.

61 Combes AN, Lefevre JG, Wilson S, Hamilton NA, Little MH: Cap mesenchyme cell swarming during kidney development is influenced by attraction, repulsion, and adhesion to the ureteric tip. Dev Biol 2016;418:297-306.

62 Mao Y, Francis-West P, Irvine KD: Fat4/ Dchs1 signaling between stromal and cap mesenchyme cells influences nephrogenesis and ureteric bud branching. Development 2015;142:2574-2585.

63 Faa G, Gerosa C, Fanni D, Monga G, Zeffanello M, Van Eyken P, Fanos V: Morphogenesis and molecular mechanisms involved in human kidney development. J Cell Physiol 2012; 227:1257-1268

$64 \mathrm{Gu}$ Y, Zhao Y, Zhou Y, Xie Y, Ju P, Long Y et al: Zeb1 is a potential regulator of Six2 in the proliferation, apoptosis and migration of metanephric mesenchyme cells. Int J Mol Sci 2016;17:pii:E1283.

65 Sims-Lucas S, Argyropoulos C, Kish K, McHugh K, Bertram JF, Quigley R, Bates CM: Three-dimensional imaging reveals ureteric and mesenchymal defects in Fgfr2-mutant kidneys. J Am Soc Nephrol 2009;20:25252533.

66 Short KM, Smyth IM: Imaging, analysising and interpreting branching morphogenesis in the developing kidney. Results Probl Cell Differ 2017;60:233-256.

67 Hokke SN, Armitage JA, Puelles VG, Short KM, Jones L, Smyth IM, Bertram JF, CullenMcEwen LA: Altered ureteric branching morphogenesis and nephron endowment in offspring of diabetic and insulin-treated pregnancy. PLoS One 2013;8:e58243.

68 Gerosa C, Fanni D, Faa A, Van Eyken P, Ravarino A, Fanos V, Faa G: Low vascularization of the nephrogenic zone of the fetal kidney suggests a major role for hypoxia in human nephrogenesis. Int Urol Nephrol 2017, Epub ahead of print.

69 Minuth WW, Denk L: Special morphological features at the interface of the renal stem/progenitor cell niche force to reinvestigate transport of morphogens during nephron induction. Biores Open Access 2016;5:49-60.

70 Oxburgh L, Muthukrishnan SD, Brown A: Growth factor regulation in the nephrogenic zone of the developing kidney. Results Probl Cell Differ 2017;60:137-164.

71 Luyckx VA, Perico N, Somaschini M, Manfellotto D, Valensise H, Cetin I, et al: A developmental approach to the prevention of hypertension and kidney disease: a report from the low birth weight and nephron number working group. Lancet 2017;390:424-428. 